\title{
Improving electric thermal stability of polypropylene by chemically linking small amount of hindered phenol groups
}

\author{
Xin Chen ${ }^{1,2} \cdot$ Wenyi Zhu ${ }^{1,3} \cdot$ Q. M. Zhang ${ }^{1,2,3}$
}

Received: 1 December 2020 / Accepted: 20 January 2021 / Published online: 3 February 2021

(c) The Author(s), under exclusive licence to The Materials Research Society 2021

\begin{abstract}
Thermal stability of polypropylene (PP) over a broad temperature is critical for many applications. Hindered phenol (HP) groups have been utilized in PP for thermal-oxidative protection. This paper studies thermal stability of the electret property of PP linked with $0.2 \mathrm{~mol} \%$ HP. It is observed that small amount of chemically linked HP groups improves electret thermal stability as reflected by the higher peak temperature of the thermally stimulated discharge curve and about $65 \%$ increase in the trap level. In addition, the HP groups in PP generate "rigid backbones" which maintain the PP film shapes to temperatures near the melting $\left(\sim 150^{\circ} \mathrm{C}\right)$, compared with pristine $\mathrm{PP}$ at $70^{\circ} \mathrm{C}$.
\end{abstract}

Keywords Polymer electrets $\cdot$ Thermal-stability $\cdot$ Polypropylene $\cdot$ Dielectric

\section{Introduction}

Polymers are ubiquitous in our society because of their unique properties, such as lightweight, flexibility, low cost, corrosion-resistance, and manufacturability. Polypropylene, as the one of the most widely used polymers in the world, is also playing critical role in polymer electrets and film capacitors. For example, N95 respirators is a key personal protective equipment (PPE) in fighting COVID-19. The heart of N95 respirators is the electrostatic polypropylene (PP) fiber air filter, which, through the surface charges, effectively traps (filters) virus and protects medical professionals and public from viral infection [1-4]. PP fiber electret filters, which are electrically charged, can retain stored charges at traps in the polymer for long period of time. Compared with

Supplementary Information The online version of this article (https://doi.org/10.1557/s43580-021-00016-1) contains supplementary material, which is available to authorized users.

Q. M. Zhang

qxz1@psu.edu

1 Materials Research Institute, The Pennsylvania State University, University Park, PA 16802, USA

2 Materials Science and Engineering Department, The Pennsylvania State University, University Park, PA 16802, USA

3 Electrical Engineering Department, The Pennsylvania State University, University Park, PA 16802, USA pure mechanical filters (such as in cloth face masks), the electrostatic filter in face masks increases the efficiency several orders of magnitude for the same pressure drop [1,3-5]. Due to the shortage of the electrostatic PP fiber filters in N95 respirators, it is recommended that general public should not use N95 respirators. One solution to address the shortage of N95 respirators is to disinfect used masks, for example, heat treating the face masks to higher than $70{ }^{\circ} \mathrm{C}$ for a given time, so that they can be reused. However, due to the thermal properties of PP electret, trapped charges will be detraped at temperatures higher than $70{ }^{\circ} \mathrm{C}[6,7]$, thus limits the cycle use of the N95 respirators.

In addition, the PP electrets are also used in piezo-sensors and transducers owing to their high piezoelectric coefficients $(>400 \mathrm{pC} / \mathrm{N}$ ) which are comparable to that of piezoceramics $[7,8]$. However, the piezo-performance of the PP electret (electrostatic) sensors and transducers deteriorates above $70{ }^{\circ} \mathrm{C}$ because of de-trapping of stored charges [7].

Hence there is a great interest in investigating and developing low cost and highly scalable approaches to improve the thermal stability of PP electret. This paper investigates a modified PP which with tiny amount of phenol groups (0.2 mol\%) chemically attached to the PP chains, see Fig. 1a for the chemical structure of PP-HP (hindered phenol), exhibits improved electric thermal performance of PP. For example, thermally stimulated depolarization current (TSDC) data shows a shift of discharge peak from $104{ }^{\circ} \mathrm{C}$ of pristine $\mathrm{PP}$ to $127^{\circ} \mathrm{C}$ of $\mathrm{PP}-\mathrm{HP}$ in addition to an increase 
(a)
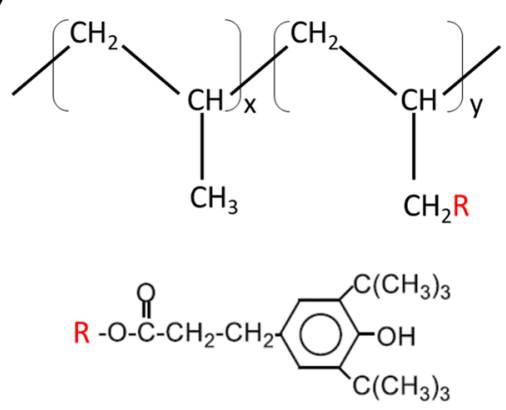

(b)

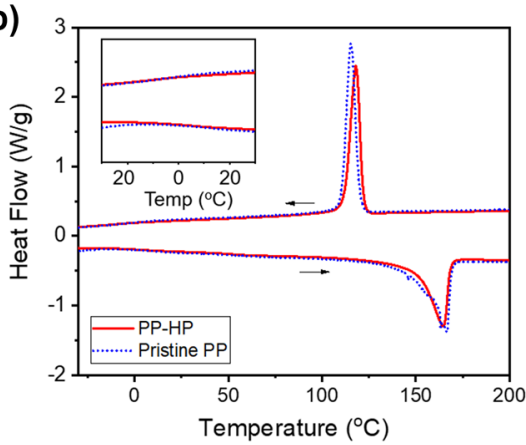

(c)

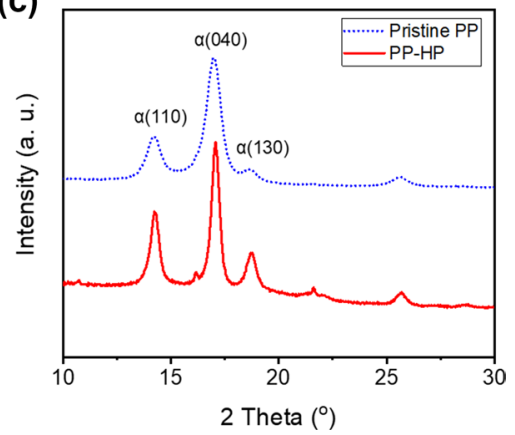

Fig. 1 a Schematic of chemical structure for modified polypropylene (PP-HP) [14]. b DSC cycles for PP-HP and pristine PP, the inset is an indication of the temperature range around glass transition for the polymers. $\mathbf{c}$ XRD of PP-HP and pristine PP

in the trap level. The thermal-mechanical properties such as polymer film shape changes with temperature are studied since it is also importance for PP fiber air filters and for electret sensors and transducers. Film shape changes, as measured by capacitance change vs. temperature, reveals that PP-HP films maintain $\Delta C / C_{25}<2.5 \%$ to $125^{\circ} \mathrm{C}$, compared with films of pristine $\mathrm{PP}$ of $75^{\circ} \mathrm{C}$, where $C_{25}$ is the capacitance at $25^{\circ} \mathrm{C}$ and $\Delta C$ is the capacitance change.

$\mathrm{PP}$ is a semicrystalline polymer, having a glass transition $T_{\mathrm{g}}$ near $0{ }^{\circ} \mathrm{C}$ and melting temperature $T_{\mathrm{m}} \sim 170{ }^{\circ} \mathrm{C}$. In general, amorphous polymer chains exhibit strong segment motions at temperatures above $T_{\mathrm{g}}$, which would facilitate charge detrapping in polymer electrets [9]. In addition, chain relaxations in polymers may cause shape changes in polymer fiber filters. Nanocomposite approach has been used to improve polymer performance, including electret performance $[8,10,11]$. It has been reported that the aromatic groups, due to their rigid molecular structure, are useful to improve the thermal stability of amorphous and semicrystalline polymers, such as in PP and Poly(ethylene naphthalate) (PEN), a better substitute for Poly(ethylene terephthalate) (PET), see Scheme S1 for the chemical structures of the polymers $[12,13]$. Therefore, chemically linking phenyl groups to PP chains may provide a solution to improve the thermal performance of PP electrets. Recently, a "PP nanocomposite", a PP containing low mol\% of phenol groups was developed by Polymics, Ltd and Advanced Polyolefin Technologies (State College, PA) [14]. On the other hand, as seen from Fig. 1a, the rigid phenol groups will not be able to co-crystallize with PP. Hence, HPs in PP-HP copolymer may cause a reduction of crystallinity. For a semicrystalline polymer with $T_{\mathrm{g}}$ near $0{ }^{\circ} \mathrm{C}$, a large reduction of crystallinity is not desired because it may cause reduction in the thermal performance of PP electrets. For example, the crystalliteamorphous interfaces are likely the defect sites for trapping charges and hence a large reduction of crystallinity will reduce the electret (charge storage) performance even at room temperature $[15,16]$. Hence, PP-HP with $0.2 \mathrm{~mol} \%$ HP, low mol\% of HP, is chosen for this study. For a comparison pristine PP films are also studied. The TGA (thermalgravimetric analysis) data, Fig. S1, reveal that $0.2 \mathrm{~mol} \%$ HP in PP-HP raises the degradation temperature to $262^{\circ} \mathrm{C}$, compared with the pristine $\mathrm{PP}$ of $230{ }^{\circ} \mathrm{C}$, a $32{ }^{\circ} \mathrm{C}$ increase.

\section{Experimental details}

PP-HP films of $20 \mu \mathrm{m}$ thick were fabricated by Polymics Ltd using extrusion method. Films of pristine PP $20 \mu \mathrm{m}$ think fabricated using extrusion method were provided by PolyK Technologies (State College, PA). The thermal properties were characterized with differential scanning calorimeter (DSC, Q100, TA) at the heating/cooling rate of $10{ }^{\circ} \mathrm{C} / \mathrm{min}$ in a nitrogen atmosphere and with TGA (TA Instruments Q600) at heating rate of $10 \mathrm{C} / \mathrm{min}$ in an air atmosphere. $\mathrm{X}$-ray diffraction (XRD) analysis was conducted using a Panalytical X'pert Pro MPD diffractometer. The wavelength of X-ray is 1.54 A. For electric characterization, Au films of $50 \mathrm{~nm}$ thick and $6 \mathrm{~mm}$ diameter were sputtered on the two surfaces of polymer films. The dielectric properties of polymer films were characterized by a HP 4294 impedance analyzer coupled to a temperature chamber. For TSDC study, the film was charged under a high voltage of $200 \mathrm{MV} / \mathrm{m}$ electric field at $100{ }^{\circ} \mathrm{C}$ for $20 \mathrm{~min}$ and then, the film was cooled down to $10^{\circ} \mathrm{C}$ under the field. The field was reduced to zero and TSDC was characterized using a HP 4140B pA meter with a heating rate of $2{ }^{\circ} \mathrm{C} / \mathrm{min}$.

\section{Results and discussion}

The DSC data presented in Fig. $1 \mathrm{~b}$ and summarized in Table 1 indicate that $0.2 \mathrm{~mol} \% \mathrm{HP}$ causes a slight reduction of $\mathrm{T}_{\mathrm{g}}$ and meanwhile the melting peak also shifts slightly to 
Table 1 Summary of several thermal properties deduced from DSC curves

\begin{tabular}{lllll}
\hline Films & $\begin{array}{l}\text { Glass transi- } \\
\text { tion temp }\left({ }^{\circ} \mathrm{C}\right)\end{array}$ & $\begin{array}{l}\text { Melt peak } \\
\text { temp }\left({ }^{\circ} \mathrm{C}\right)\end{array}$ & $\begin{array}{l}\text { Melt } \\
\text { enthalpy } \\
(\mathrm{J} / \mathrm{g})\end{array}$ & $\begin{array}{l}\text { Estimated } \\
\text { crystallin- } \\
\text { ity }\end{array}$ \\
\hline Pristine PP & 3.5 & 166.45 & 87.09 & $54.1 \%$ \\
PP-HP & 1.5 & 163.62 & 81.44 & $50.6 \%$ \\
\hline
\end{tabular}

lower temperature than that of pristine PP. The crystallinity of PP-HP is estimated by using the melt enthalpy divided by the standard enthalpy of $100 \%$ crystallinity PP, i.e., $161 \mathrm{~J} / \mathrm{g}$ [17]. The crystallinity of PP-HP is $50.6 \%$, a slight decrease from $54.1 \%$ of pristine PP. The dielectric data in Fig. 2a shows that the PP-HP ( $0.2 \mathrm{~mol} \%)$ maintains the dielectric properties of pristine PP, e.g., the dielectric constant $K=2.25$ and dielectric loss $<0.002$.

Early dielectric spectroscope studies of PP revealed two dielectric relaxation processes, a dielectric relaxation at low temperature is associated with the glass transition ( $\alpha$ relaxation) and the high temperature one $\left(\sim 100^{\circ} \mathrm{C}\right)$ is associated with crystallization ( $\beta$ relaxation) [18]. TSDC is carried out to probe the depolarization peak and trap depth for the polymers associated with the high temperature polymer chain relaxation which is of interest of this study [19]. As shown in Fig. 2b, the discharge peak shifts from $104{ }^{\circ} \mathrm{C}$ of pristine PP to $127{ }^{\circ} \mathrm{C}$ of $\mathrm{PP}-\mathrm{HP}$, a raise of $23{ }^{\circ} \mathrm{C}$. The results suggest that the rigid phenol groups in PP-HP, even at $0.2 \mathrm{~mol} \%$ level, impede polymer chain motions and raise the depolarization temperature by more than $20^{\circ} \mathrm{C}$.

For polymer electrets, the trap depth $E_{\mathrm{t}}$ is also a critical parameter. In electrets, charge detrapping is a thermally activated process, $\sim \exp \left(-E_{\mathrm{t}} / k_{\mathrm{b}} T\right)$, where $\mathrm{k}_{\mathrm{b}}$ is the Boltzmann's constant and $\mathrm{T}$ is the absolute temperature. At room temperature $k_{\mathrm{b}} T=0.025 \mathrm{eV}$. Hence traps with larger $E_{\mathrm{t}}$ can maintain stored charges to higher temperatures. The trap depths can be estimated from the TSDC peaks [19]. In most studies, the initial rising slope is used to deduce trap energy level $E_{\mathrm{t}}$, e.g., [19]

$\ln (I(T))=$ constant $-\frac{E t}{k t}$

The trap depth deduced for the pristine PP is $0.3 \mathrm{eV}$, which is similar to what has been reported for PP, [20] and for PP-HP, it is $0.51 \mathrm{eV}$. Hence, $0.2 \mathrm{~mol} \% \mathrm{HPs}$ in PP-HP generate deeper trap than the pristine $\mathrm{PP}$ which can provide better retention of stored charges or maintaining the same charge retention at higher temperatures. The integrated area of the discharge peaks provides a comparison of trapped charges between PP-HP and pristine PP films: the charges released in the peak is $6.77 \times 10^{-9} \mathrm{C}$ for pristine $\mathrm{PP}$ and $6.47 \times 10^{-9} \mathrm{C}$ for PP-HP. These results show that $0.2 \mathrm{~mol} \%$ HPs in PP-HP can enhance the thermal stability of the PP electret for more than $20^{\circ} \mathrm{C}$ which is attractive for the modified PP polymer for use in the PP electrostatic fiber filters and for PP electret sensors and transducers.

To understand the molecular origin of the enhanced trap levels in PP-HP (0.2 mol\%), we also carried out x-ray diffraction study of the PP-HP copolymer, which is presented in Fig. 1c. As shown in Fig. 1c, with such small amount of chemical additive, the polymer does not exhibit obvious changes in crystal structures, the diffraction peaks reveal the major presence of $\alpha$ phase [17,21]. Since there is not much change in DSC and XRD data between pristine PP and PP-HP, the results suggest that the enhanced trap depth is mainly caused by the HP groups. Charge traps can form at crystalline-amorphous interfaces and at chemical defects. If the PP-HP is treated as "a nano-composite", interface regions surrounding the HP groups may generate deeper traps than that in the pristine PP due to the hinderance effect of the phenol groups. In our recent study of polymer nanocomposites, it was observed that in PEEU, very low volume loading of nanofillers ( $\sim 0.2 \mathrm{vol} \%)$ can generate deeper traps
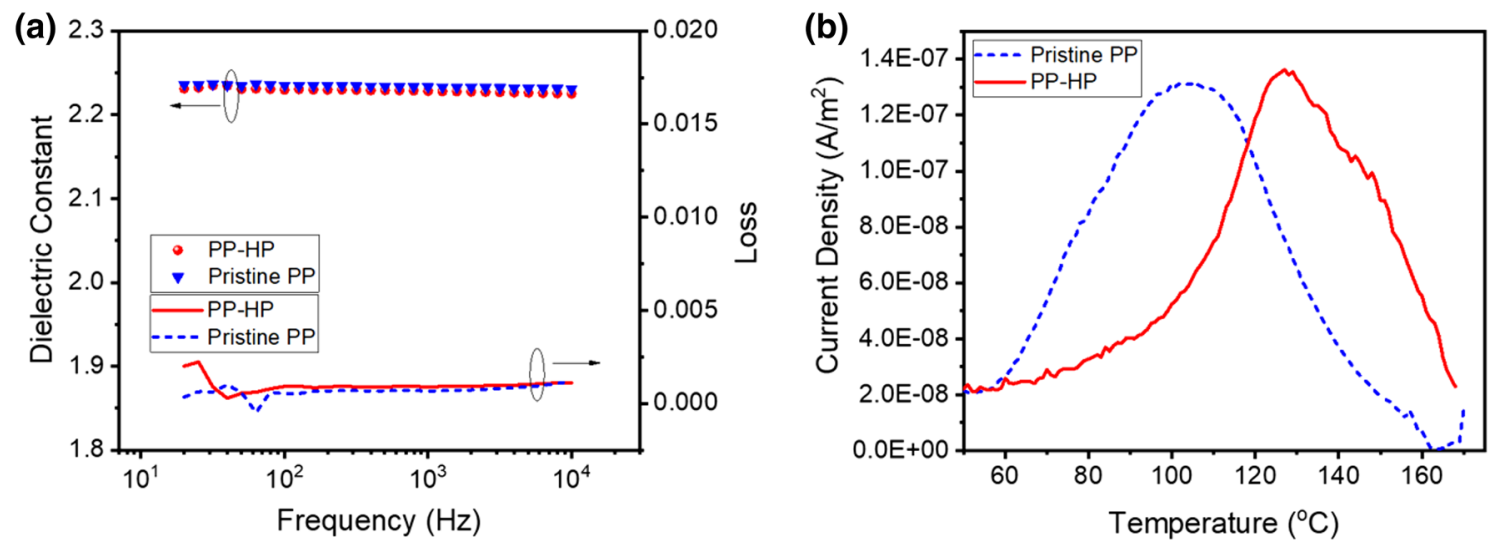

Fig. 2 a Dielectric constant and loss vs. freq. at room temperature and b TSDC curves for PP-HP and PP 
(a)

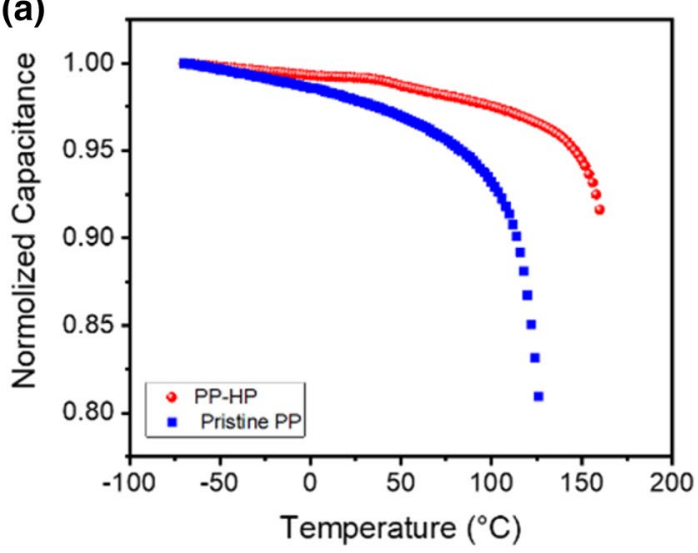

(c)

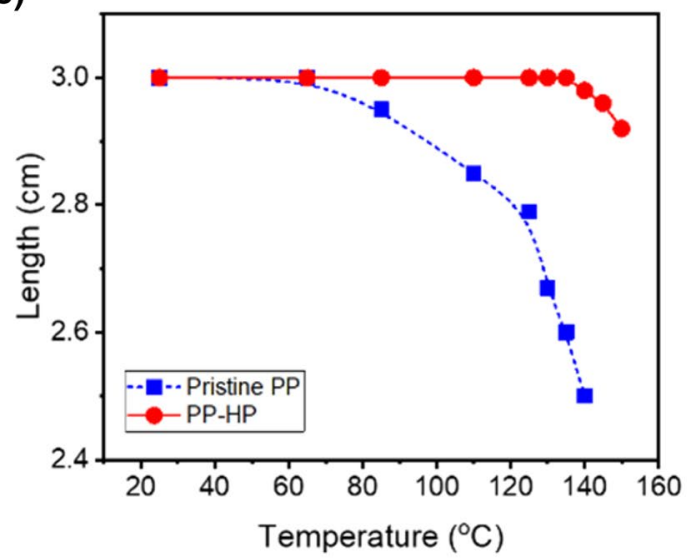

(e)

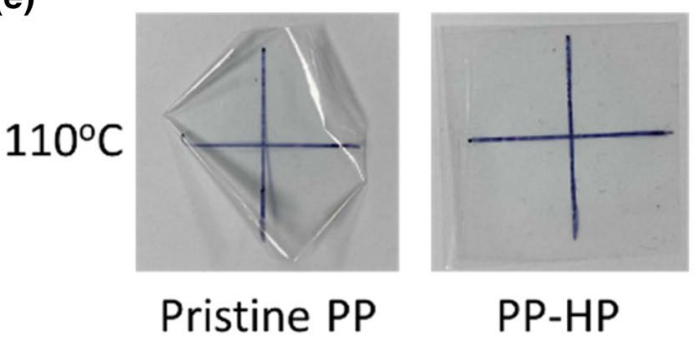

Fig. 3 a Temperature dependent normalized capacitance for pristine $\mathrm{PP}$ and PP-HP from experiment. b Temperatures at which the relative capacitance changes $\Delta C / C_{25}$ where $C_{25}$ is the capacitance at $25^{\circ} \mathrm{C}$ are $10 \%$ for pristine PP and PP-HP. c The length of marked lines

in the nanocomposites, compared with pristine polymers [22].

For polymer electrostatic fiber air-filters, shape changes of PP fibers due to high temperature treatment, for example, high temperature disinfection, may affect the performance. To evaluate the thermal-mechanical properties, the measurement of capacitance changes with temperature is carried out to provide the shape changes of PP-HP and pristine PP. Such kind of measurement is widely used in capacitor industry since a large change of capacitance with

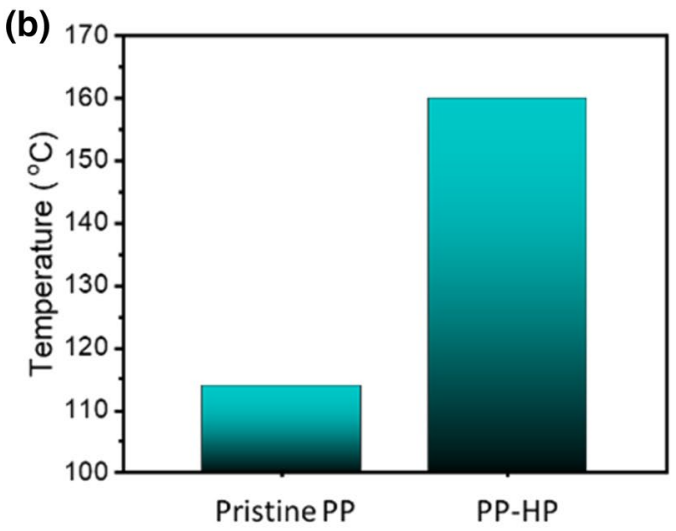

(d)
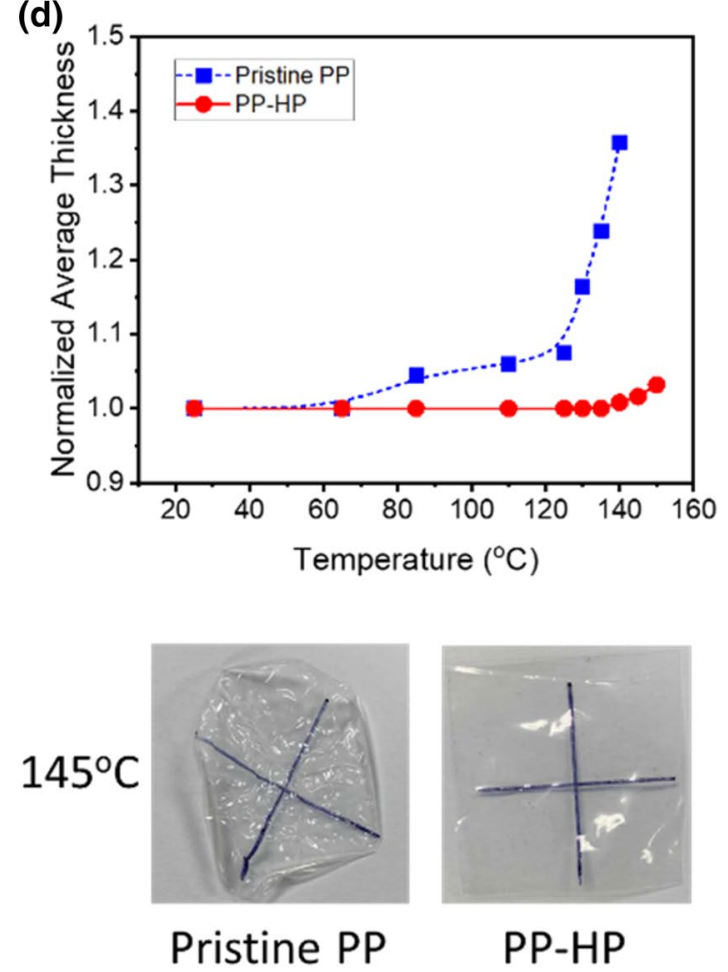

(as indicated in figure S3) vs. temperature, $\mathbf{d}$ normalized thickness change vs. temperature for pristine $\mathrm{PP}$ and $\mathrm{PP}-\mathrm{HP}$, e Shape changes of pristine PP and PP-HP films at $110^{\circ} \mathrm{C}$ and $145^{\circ} \mathrm{C}$

temperature is not desired for most electric and electronic devices and systems. For film capacitors, the capacitance $\mathrm{C}$ is

$C=\frac{K \varepsilon_{0}}{d} A$

where $\varepsilon_{0}\left(=8.85 \times 10^{-12} \mathrm{~F} / \mathrm{m}\right)$ is the vacuum permittivity, $A$ is the electrode area and $d$ is the film thickness. Any changes in film area $A$ and thickness $d$ due to temperature generated shape change as well as change of $K$ with temperature will 
Table 2 Ratio of capacitance change $\Delta C / C_{25}$ at different temperatures where $C_{25}$ is the capacitance at $25^{\circ} \mathrm{C}$

\begin{tabular}{lllll}
\hline Films & $50{ }^{\circ} \mathrm{C}$ & $100{ }^{\circ} \mathrm{C}$ & $130{ }^{\circ} \mathrm{C}$ & $150{ }^{\circ} \mathrm{C}$ \\
\hline PP & $-1.1 \%$ & $-4.9 \%$ & $-22.5 \%$ & $-60.5 \%$ \\
PP-HP & $-0.5 \%$ & $-1.7 \%$ & $-2.9 \%$ & $-4.8 \%$ \\
\hline
\end{tabular}

cause change in capacitance. For PP, the refractive index n is reported in the range from 1.49 to 1.52 [23]. As shown in Fig. 2a, the PP and PP-HP films have $K=2.25$ at room temperature which is nearly equal to $n^{2}$. Hence, for PP, the $K$ is almost all from the electronic contribution, i.e., $K=n^{2}$. In the literature, it has been reported that the dielectric constant of PP films will decrease $1 \%$ at $70{ }^{\circ} \mathrm{C}, 3 \%$ at $100{ }^{\circ} \mathrm{C}$, and $6 \%$ at $120{ }^{\circ} \mathrm{C}$ [24].

The capacitance changes of the two types of PP films vs. temperature are presented in Fig. 3 a, measured in temperature from -75 to $160{ }^{\circ} \mathrm{C}$. PP-HP exhibits exceptionally high temperature stability of capacitance, mainly due to its high thermal stability of the film shape (as indicated in Fig. S2 for films with Au electrodes). For example, as shown in Table 2. PP-HP films maintain the capacitance change less than $5 \%$ at temperatures up to $150{ }^{\circ} \mathrm{C}$ which is very close to the melting temperature of PP. In comparison, at $100{ }^{\circ} \mathrm{C}$, PP films exhibit capacitance changes larger than 5\%. The temperatures for $10 \%$ capacitance change for these two polymers are compared in Fig. 3b.

The dimension changes of the polymer films such as the thickness of the films and the lateral dimension as marked cross on the surfaces of the polymer films at various temperatures are also directly measured. The initial length (at $25^{\circ} \mathrm{C}$ ) marked cross is $3.0 \mathrm{~cm}$ (See Fig. S3). The film thickness was measured by Heidenhain Length Gauges from Powertronics Incorporated. As shown in Fig. S3, the shape of the pristine PP films displays obvious change at $85^{\circ} \mathrm{C}$. At temperatures higher than $110^{\circ} \mathrm{C}$, pristine PP cannot retain its shape and undergo a large shrinkage. In contrast, the PP-HP possesses much better shape stability at higher temperatures compared to the pristine PP. At $150{ }^{\circ} \mathrm{C}$, the shape of PP-HP is still similar to that at room temperature. The dimension changes for these polymer films are presented in Fig. $3 \mathrm{c}$ and d (deduced from the images shown in Fig. S3), shrinking of the films lead to the increase of thickness and decrease of the film area. It can be seen that the length $(L)$ and thickness of PP-HP do not exhibit significant change with temperature even at $150{ }^{\circ} \mathrm{C}$. We note that improved thermal-mechanical properties such as shape stability of PP have been observed in PP/carbon nanofiber (CNF) composites. It is suggested the CNFs can restrict the motion of polymer chains, thus reducing the tensile strain stimulated by temperature [25]. For PP-HP, the HP groups in the PP polymer form stiff mechanical backbones (frames) which maintain the polymer film shape stability at temperatures close to $T_{\mathrm{m}}$ of PP.

\section{Conclusion}

In summary, this study finds that PP-HP with only $0.2 \mathrm{~mol} \%$ HP can lead to improvement of temperature stability of the electret charge storage performance as reflected by the higher peak temperature of TSDC curve and more than $65 \%$ increase in the trap level. The HP groups in PP-HP cause small reduction of crystallinity, about $6 \%$, which may be responsible for the slight decrease of the charges under the TSDC peak, about $4.5 \%$, of PP-HP compared with pristine PP. It is also observed that the PP-HP films maintain their shapes to temperature near the melting $\left(150^{\circ} \mathrm{C}\right)$, compared with pristine PP starting to deform at $70^{\circ} \mathrm{C}$, suggesting that the HP groups form "rigid backbones" in PP-HP.

Acknowledgments We thank Polymics, Ltd for providing the PP-HP films and PolyK Technologies LLC for providing Pristine PP films. We also thank Dr. Minren Lin for discussions. This material is based upon that reflected the research supported by the Office of Naval Research under Award Number N00014-19-1-2028.

\section{References}

1. R.C. Brown, Air Filtration: An Integrated Approach to the Theory and Applications of Fibrous Filters (Pergamon Press, Oxford, 1993).

2. J. Van Turnhout, W.J. Hoeneveld, J.W.C. Adamse, L.M. Van Rossen, IEEE Trans. Ind. Appl. 17(2), 240-248 (1981)

3. R. Thakur, D. Das, A. Das, Sep. Purif. Rev. 42, 87-129 (2013)

4. C.S. Wang, Powder Technol. 118, 166-170 (2001)

5. J. Xiao, J. Liang, C. Zhang, Y. Tao, G.-W. Ling, Q.-H. Yang, Small Methods 2, 1800012 (2018)

6. R. Fischer, D.H. Morris, N. van Doremalen, S. Sarchette, J. Matson, T. Bushmaker, C.K. Yinda, S. Seifert, A. Gamble, B. Williamson, S. Judson, E.D. Wit, J. Lloyd-Smith, and V. Munster, Assessment of N95 respirator decontamination and re-use for SARS-CoV-2. medRxiv (the preprint server for health sciences).

7. G.S. Neugschwandtner, R. Schwodiauer, S. Bauer-Gogonea, S. Bauer, J. Appl. Phys. 89, 4503-4511 (2001)

8. S. Bauer, IEEE Trans. DEI 13, 953 (2006)

9. R.J. Young, P.A. Lovell, Introduction to Polymers (CRC Press, New York, 2011).

10. C. Huang, Q.M. Zhang, J.Y. Li, M. Rabeony, Appl. Phys. Lett. 87, 182901 (2005)

11. B.D. Chen, W. Tang, C. Zhang, L. Xu, L.P. Zhu, L.J. Yang, C. He, J. Chen, L. Liu, T. Zhou, Z.L. Wang, Nano Res. 11, 3096 (2018)

12. M.W. Sabaa, E.H. Oraby, A.S. Abdel-Naby, R.R. Mohamed, Polym. Degrad. Stab. 91, 911-923 (2006)

13. A.E. Tonelli, Polymer 43, 637-642 (2002)

14. G. Zhang, C. Nam, L. Petersson, J. Jambeck, H. Hillborg, T.C. Mike Chung, Macromolecules 51, 1927-1936 (2018)

15. G.M. Sessler, Physical principles of electrets, in Electrets. (Springer, Berlin, 1980), pp. 13-80 
16. L. Chen, T.D. Huan, R. Ramprasad, Sci. Rep. 7, 1-10 (2017)

17. R. Krache, R. Benavente, J.M. Lopez-Majada, J.M. Perena, M.L. Cerrada, E. Pérez, Macromolecules 40, 6871-6878 (2007)

18. T. Umemura, T. Suzuki, T. Kashiwazaki, IEEE Trans. Elect. Insulat. 17(4), 300 (1982)

19. S. Maeta, K. Sakaguchi, Jpn. J. Appl. Phys. 19, 519 (1980)

20. T. Iqbal, C.A. Hogarth, Thin Solid Films 61, 23-26 (1979)
21. E. Assouline, E. Wachtel, S. Grigull, A. Lustiger, H.D. Wagner, G. Marom, Polymer 42, 6231-6237 (2001)

22. T. Zhang, X. Chen, Y. Thakur, B. Lu, Q. Zhang, J. Runt, Q.M. Zhang, Sci. Adv. 6, eaax6622 (2020)

23. A.E. Belal, A.A. Hamza, T.Z.N. Sokkar, K.A. El-Farahaty, K.M. Yassien, Polym. Testing 21, 877-882 (2002)

24. J.L. Nash, Polym. Eng. Sci. 28, 862-870 (1988)

25. A. Chatterjee, B.L. Deopura, J. Appl. Polym. Sci. 100, 3574-3578 (2006) 